\title{
Correction: Design of Transparent Multicolor LED Signage with an Oxide-Metal-Oxide Interconnect Electrode
}

\author{
[J. Korean Phys. Soc. 77, 82 (2020)] \\ DOI: $10.3938 / \mathrm{jkps} .77 .82$ \\ JeeYeon PARK, Hyunjee JEON, Nayeon PARK and Geonwook YoO* \\ School of Electronic Engineering, Soongsil University, Seoul 07027, Korea \\ Chul Jong HAN and Min Suk $\mathrm{OH}^{\dagger}$ \\ Display Research Center, Korea Electronic Technology Institute, Seongnam 13509, Korea \\ Byeong-Kwon Ju \\ Display and Nanosystem Laboratory, College of Engineering, Korea University, Seoul 136-713, Korea \\ Yoon-su KIM \\ Display Research Center, Korea Electronic Technology Institute, Seongnam 13509, Korea and \\ Display and Nanosystem Laboratory, College of Engineering, Korea University, Seoul 136-713, Korea
}

DOI: $10.3938 / \mathrm{jkps} .77 .270$

This paper was published with a typographical error in Acknowledgments on page 86. The grant number NRF2019M3C1B909055 should read NRF-2019M3C1B9090559.

${ }^{*}$ E-mail: gwyoo@ssu.ac.kr
${ }^{\dagger}$ E-mail: ohms@keit.re.kr 